Check for updates

Cite this: Chem. Soc. Rev., 2020, 49,6568

Received 9th June 2020

DOI: $10.1039 / \mathrm{d} 0 \mathrm{cs} 00363 \mathrm{~h}$

rsc.li/chem-soc-rev

\title{
Bioinspired light-driven soft robots based on liquid crystal polymers
}

\author{
M. Pilz da Cunha, (D) M. G. Debije (D) and A. P. H. J. Schenning (DD*
}

\begin{abstract}
Nature is a constant source of inspiration for materials scientists, fueling the dream of mimicking life-like motion and tasks in untethered, man-made devices. Liquid crystalline polymers (LCPs) programmed to undergo three-dimensional shape changes in response to light are promising materials for fulfilling this dream. The successful development of autonomous, highly controlled light-driven soft robots calls for an understanding of light-driven actuation, advancements in material function and performance, and progress in engineering principles for transforming actuation into life-like motions, from simple bending to walking, for example. This tutorial review includes an introduction to liquid crystal (LC)-based materials and highlights developments in light-responsive LC polymers, shape programmability and sustained motions to finally achieve bioinspired untethered soft robots able to perform locomotion and tasks.
\end{abstract}

\section{Key learning points}

1. An overview of the properties of LCPs as stimuli-responsive materials, presenting insights into how external stimuli can trigger complex shape changes. 2. Present knowledge on the mechanistic translation of light absorption into macroscopic shape changes in LCPs and the role of molecular photoswitches. Also, highlight the challenges in the translation of simple motions, such as bending, into more complex motion, such as walking.

3. Discuss significant breakthroughs and provide illustrative examples of the current status of light-responsive LC-based materials.

4. Illustrate the importance of advancements in complementary areas ranging from fundamental understanding of light-responsive LC-based materials to engineering of actuator assemblies to prepare bioinspired untethered soft robotic devices.

5. Identify challenges facing the successful development of light-driven soft robots and highlight the necessity for multi-disciplinary collaborations in the advancement of this field.

\section{General introduction}

Admiration for the elegance of nature has fueled the dream of developing man-made materials and machines that mimic the abilities and adaptability of living creatures. Through evolution, animals have become highly functional and efficient, utilizing environmental stimuli to trigger adaptive behaviors that help the species survive. As examples, consider the multi-tasking abilities of locomotion and transportation of cargos by ants, amphibians adapting to both dry and aquatic environments and soft marine organisms with their versatility of motions.

Soft robotics help us progress towards this dream. In contrast to hard robots, in which stiff segments limit the adaptability and versatility of devices, soft robots fully composed of compliant polymers better emulate living, soft-bodied organisms and are

Laboratory of Stimuli-responsive Functional Materials \& Devices, Department of Chemical Engineering and Chemistry, Eindhoven University of Technology, P.O. Box 513, 5600 MB, Eindhoven, The Netherlands.E-mail: a.p.h.j.schenning@tue.nl more suitable for interaction with humans. To free soft robots from tethering to external or heavy on-board control units, stimuli-responsive polymers which undergo macroscopic deformations in response to remote triggers are of great interest. ${ }^{1}$ In particular, light and magnetic fields have emerged as the most popular stimuli for controlling these mechanical deformations, ${ }^{2}$ offering remote, non-destructive and precision actuation methods, with light having the advantage of not requiring bulky external magnetic setups.

Exciting developments in light-responsive liquid crystal polymers (LCPs) have highlighted the potential of these materials in untethered soft robotics. ${ }^{3}$ Even though light-driven, LCP soft robots have been reported, the majority of examples are single component devices comprised of a single film, only active in either a dry or an aqueous environment and capable of performing only a single task, or unidirectional locomotion. Expansion towards multitasking soft robots, with locomotion as well as cargo transportation in a single device, and adaptability to diverse and changing environments are research goals which, if achieved, have the 
potential to revolutionize fields such as microfluidics and biomedicine. Realizing untethered soft robots calls for advancement in three areas: (1) understanding of fundamental photoactuation mechanisms, (2) design of novel advanced materials capable of sustained motion and programmable shape changes, and (3) the construction of communicating and orchestrated assemblies which can transform simple actuation modes, such as bending, into useful actions like walking, Fig. 1.

Light-responsive polymers ${ }^{4}$ and untethered soft robotics ${ }^{5}$ are both fast-moving research fields. This tutorial review bridges these two rapidly evolving topics, fully focusing on light-driven LCP soft robots to elucidate the most relevant breakthroughs in each of the research areas illustrated in Fig. 1, as well as presenting the most advanced light-driven, centimeter-scale devices. This tutorial serves as an inspiring critical overview of current research contributions and as an accessible instruction for researchers considering entering the exciting field of LC-based soft robotics.

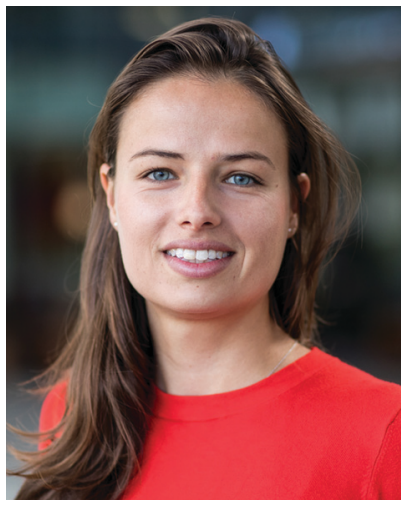

M. Pilz da Cunha

Marina Pilz da Cunha obtained her BSc and MSc degree at the Eindhoven University of Technology with research stays at the University of Porto (PT) and at the Queensland University of Technology (AU). She is currently pursuing her PhD at the Eindhoven University of Technology under supervision of Prof. Albert Schenning. Her work focuses on the development and understanding of stimuli-responsive liquid crystal polymers and their employment in soft robotic devices.

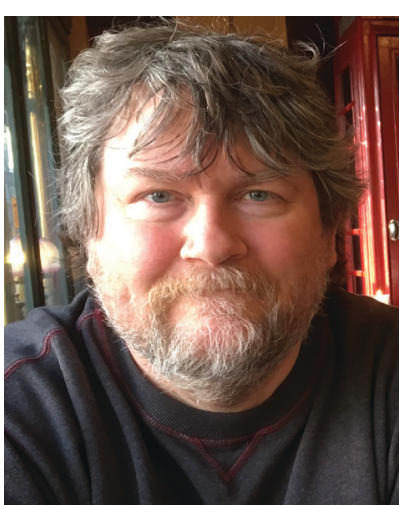

M. G. Debije
Michael Debije received a MSc in High Energy Physics from Iowa State University (USA) in 1994. After a year of teaching in Frydek-Mistek in the Czech Republic, in 2000 he went to the University of Rochester (USA) where he received a PhD in Biophysics for his study on DNA. Continuing to The Netherlands for a postdoc position at the Interfaculty Reactor Institute at $T U$ Delft in the group of John Warman, he studied charge transport in liquid crystalline discotics and organometallics. In 2003 he joined the Eindhoven University of Technology where he is currently an Assistant Professor.

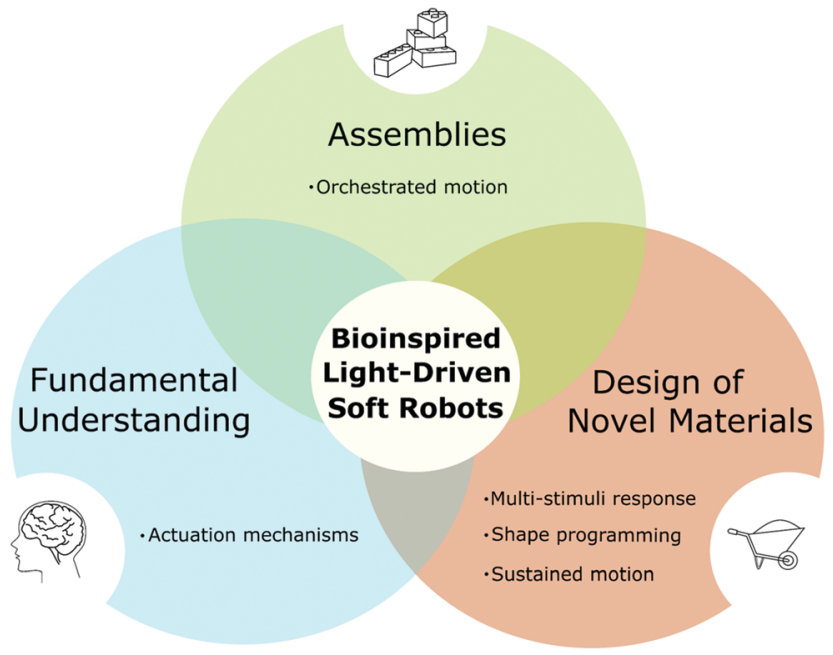

Fig. 1 Schematic diagram representing complementary areas of development which are of importance for the fabrication of bioinspired lightdriven soft robots.

\section{On light-responsive liquid crystal polymer actuators}

Stimuli-response aids in the survival of organisms; think of heliotropism, in which plants mechanically deform to track sunlight. Polymers with tunable molecular organization based on liquid crystals (LCs) have been used to fabricate materials showing macroscopic deformation to remote triggers such as light. ${ }^{6}$ LCPs are ideal materials because of their ease of lightinduced actuation in dry and wet environment and the ability to engineer molecular alignments within the LC-matrix.

\subsection{Liquid crystal-based polymers}

LC materials have physical properties between that of ordered crystalline solids and isotropic liquids. When in the LC state,

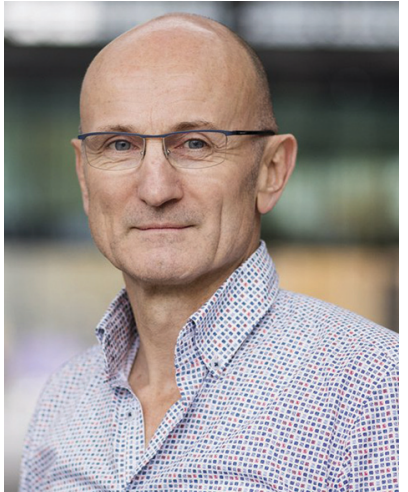

A. P. H. J. Schenning
Albert Schenning studied chemistry at Radboud University Nijmegen, where he obtained his doctorate in 1996. His PhD thesis was supervised by $\mathrm{Dr}$ Martin Feiters and Prof. Roeland Nolte. In 1996, Schenning was also a post-doctoral fellow in the group of Prof. Bert Meijer at Eindhoven University of Technology (TU/e), working on dendrimers. In 1997, he investigated oligo(triacetylene)s with Prof. François Diederich at the ETH in Zurich. From 1998 until 2003, Schenning was a fellow of the Royal Netherlands Academy of Arts and Sciences (KNAW). Currently, he is full professor of Stimuli-responsive Functional Materials and Devices at TU/e. 
these materials maintain the fluid-like ability to flow while preserving a degree of molecular order. Typical geometries of LC molecules include rod-like, disc, or bent-core. Thermotropic rod-like LCs are the prevailing class of materials used in the fabrication of light-responsive LCPs, owing to the ease of using temperature to tune the degree of molecular order in the polymer and the easy control of molecular alignments. Rod-like LC molecules typically have a rigid core with flexible aliphatic tails which can contain polymerizable end-groups, Fig. 2A. At high temperatures, such thermotropic LCs have a reduced molecular order, and are in the isotropic, liquid phase; lowering the temperature leads to molecularly ordered phases such as the smectic $(\mathrm{Sm})$ or nematic $(\mathrm{N})$. In the smectic phase, the rod-like molecules are aligned in layered structures, with the long axis of the molecules, indicated by the molecular director, pointing in a common direction. In the nematic phase, molecules lose their positional order and only retain a common orientational order. By adjusting the length of the aliphatic tails or the rigid molecular core of LC molecules and/or by using mixtures, the LC phases can be tuned. Application of electric or magnetic fields or use of surface alignment layers allows control over the orientation of the molecular director in LC phases. Such induced alignments can be fixed by (photo)polymerization if the rod-like LC molecules contain polymerizable end-groups, Fig. 2A. Polymerization results in a polymeric freestanding film. Depending on the crosslink density, LC elastomers (LCEs) or networks (LCNs) are obtained; LCEs have low crosslink densities, hence glass transition temperatures, $T_{\mathrm{g}}$, typically below room temperature, and so behaving as rubbers, while LCNs have a $T_{\mathrm{g}}$ above room temperature, and behave as glassy solids at ambient conditions. ${ }^{7}$ LCNs typically consist of (meth)acrylate-based monomers, crosslinked through one-step photopolymerization popularly carried out in a glass cell, which allows for the use of alignment layers to direct the molecular organization. LCEs, however, can have a flexible backbone, such as siloxane units, giving the material viscous properties at ambient conditions,

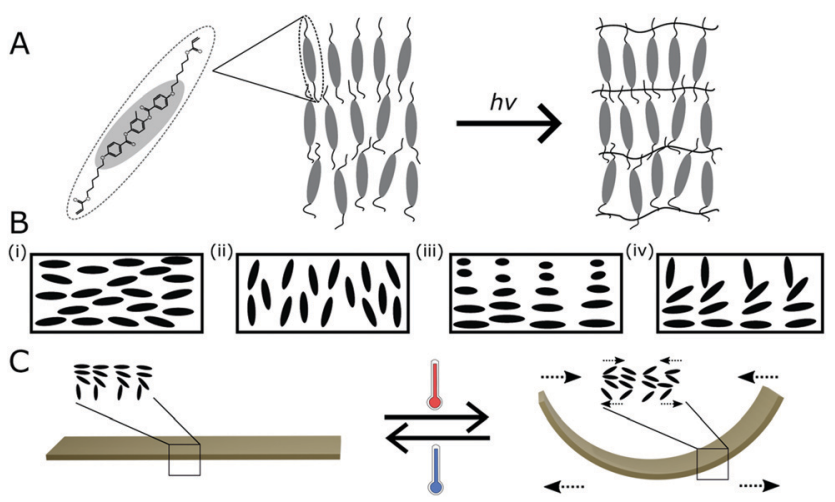

Fig. 2 (A) Schematic representation of the photopolymerization of thermotropic rod-like, diacrylate LC molecules into a crosslinked, aligned network. (B) Different molecular alignment of nematic LCs: (i) planar, (ii) homeotropic, (iii) twisted nematic and (iv) splay. (C) Macroscopic, reversible deformation of a splay aligned LCN upon heating and cooling as a result of the anisotropic thermal expansion coefficients whose effects are amplified by the discontinuous splay alignment. preventing the use of cell setups, so that LCEs are usually aligned through mechanical strain (stretching). LCEs are generally fabricated through a two-step crosslinking technique developed by Finkelman, ${ }^{8}$ in which the LC mesogens are first slightly crosslinked, allowing for the application of mechanical strain to induce molecular orientation, followed by a final crosslinking step to form the rubber-like material.

Whereas LCEs often have a continuous alignment, i.e. along the stretching direction, LCN polymers may have uniform or nonuniform alignments through the thickness of the film, Fig. 2A. Two common uniform alignments are planar and homeotropic: the LC molecules are uniformly oriented either parallel or perpendicular to the film surfaces, respectively. More complex, non-uniform alignments include splay or twisted nematic, with splay being a gradual transition between planar and homeotropic through the sample depth, while in twisted nematic the molecular planes are rotated through the sample thickness. The resulting polymerized networks display anisotropic properties: of special interest for actuators are the mechanical and thermal expansion anisotropies. A direct effect of molecular and orientational anisotropy can be observed when heating planar aligned LCNs and LCEs: due to a slight decrease in the molecular order contraction takes place along the long axis of the molecules, and expansion perpendicular. For LCNs with continuous planar or homeotropic alignments, such thermally driven deformations will not be obvious at the macroscopic scale, with shrinkage and expansions of typically less than $1 \% .^{9}$ However, thermally driven deformations can be amplified by utilizing the non-uniform alignments such as splay, in which the different molecular orientation of the two sides of the film amplify the anisotropic volume expansions and lead to larger macroscopic deformations, Fig. 2B. The mechanism for actuation in ordered LCE materials differs from LCNs, in that the polymer network does not maintain its orientation and alignment when heated: instead, it undergoes a transition to the isotropic phase, disrupting the anisotropy of the polymer network. This phase transition leads to a large uniaxial contraction of up to $400 \%$ along the nematic director; subsequent cooling reverses the deformation and the film may return to its initial shape. ${ }^{10}$ Even though LCEs also actuate below the $T_{\mathrm{NI}}$ due anisotropic expansion coefficients, the dominant actuation mechanism follows from the large disorder brought by the phase transition.

\subsection{Understanding light-driven liquid crystal actuators}

Incorporation of molecular photoswitches into LCPs allows for the translation of absorbed light energy into mechanical work. Photosensitive molecules including spiropyrans, hydrazones, and azobenzene derivatives have been explored in LCPs, with the latter being the most popular due to easy integration into the polymer without extensive disruption of the molecular alignment. ${ }^{11}$ In regards to LCPs, photosensitive dyes can be categorized in two groups which classify their primary mechanism for photoactuation: photothermal and photomechanical photoswitches. The nature of the employed photosensitive dye is of great importance, influencing the speed of actuation, reversibility of the direction of motion, and determines whether actuation is possible in dry as well as aqueous environments. 
A Photothermal photoswitch (i)

(ii)

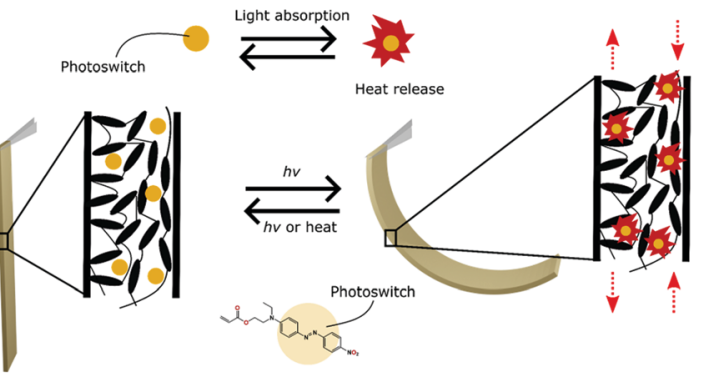

B Photomechanical photoswitch

(i)

(ii)

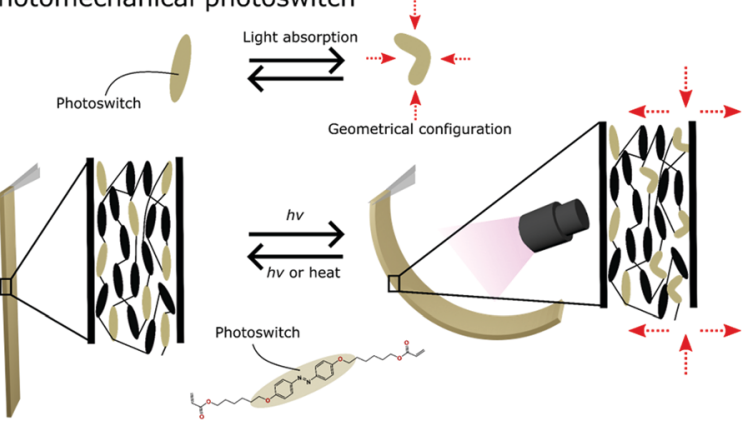

Fig. 3 Photoswitches in LCPs. (A) Photothermal photoswitch doped into an LCP with splay network. Photothermal photoswitches are doped in the polymer with or without connection to the network. Upon illumination, the chromophore absorbs light and releases heat (i), which is dissipated through the network. The photoswitch presented in (i) is a monoacrylate azobenzene derivative. Actuation is a result of anisotropic thermal expansion (ii). (B) Photomechanical photoswitch doped into an LCP. Photomechanical photoswitches can be employed as crosslinkers or as part of the polymer main-chain. Upon illumination the photoswitch undergoes a change in its geometry, (i), such as isomerization from trans to cis isomer. The photoswitch presented in (i) is a diacrylate azobenzene derivative. When doped into a planar aligned network at high concentrations, the isomerization of these crosslinker chromophores can lead to the bending of the film towards the light source (directed from the right). Actuation is a result of the strain gradient caused by the 'network pull' caused by the cis state isomers on the irradiated surface of the film, (ii).

Photothermal dyes function by transforming absorbed light into heat, which is dissipated through the polymer, Fig. 3A(i). The released heat will result in macroscopic actuation which follows a similar mechanism to the thermal deformation discussed in the previous section. ${ }^{12}$ For LCEs, the photothermal deformation will be rooted in phase transitions, while for LCNs on the amplification of thermal expansions by discontinuous alignments, such as splay or twisted nematic. Photothermal agents need not be crosslinked into the network and can be dispersed as dopants, similar to graphene, gold nanoparticles or carbon nanotubes, Fig. 3A(ii). Commonly used photosensitive dyes in LCPs, such as azobenzenes, can also cause fully photothermal-driven actuation when doped in the network as side-chain molecules. ${ }^{13}$ Often donor-acceptor substituted azobenzenes are used as photothermal agents due to the short cis isomer half-life time. An additional effect inherent in photothermal dyes is the network photo-softening responsible for the light-induced storage modulus decrease, which is significantly larger than the thermal softening of glassy polymers commonly observed. By decreasing the network's storage modulus, photosoftening can contribute to enhanced actuation. Photothermal chromophores can cause fast actuation which is immediately reversed upon switching off the excitation light. However, such chromophores limit the actuation of LCPs to dry environments, since when actuated underwater, dissipation of heat to the surrounding medium severely inhibits motion. Recent work has shown underwater actuation of a very soft LCP is possible, but only by using very high laser intensities to achieve sufficient heating. ${ }^{14}$

Photomechanical photoswitches, on the other hand, are often incorporated into LCPs as crosslinkers or as part of the main-chain in LCEs. Upon light absorption, these chromophores undergo molecular scale motion, typically isomerization (Fig. 3B(i)), which subsequently causes macroscopic motion (Fig. 3B(ii)). Upon illumination, dyes such as crosslinker azobenzenes exert a force on the surrounding network via light-triggered geometrical changes in its structure, from the thermodynamically stable rod-like shaped trans isomer, into its relatively long-lived bent cis state. ${ }^{15}$ The force is referred to as the 'network stress' or 'pull effect', and results in disruption of molecular order, causing a volumetric contraction along the molecular director and an expansion perpendicular. Another photomechanical effect is free-volume formation, representing the changes in molecular volume in the immediate vicinity of the photoswitch during isomerization. Whereas most reports investigate the actuation of azobenzene-containing LCPs upon exposure to UV light, irradiation with polarized blue/green light has also shown to causes actuation through photoreorientation, termed trans-cistrans reorientation or Weigert effect, due to the nearly equivalent absorption of trans and cis isomers at these wavelengths. ${ }^{16}$ However, such effects has been shown to generate lower strains than more often employed trans-cis isomerization. ${ }^{17}$

When actuated in dry environments, photomechanical photoswitches may also release heat, resulting in a combination of thermal and mechanical contributions to actuation in dry environments. Decoupling of photomechanical effects from temperature allows LCPs containing photomechanical chromophores to act as amphibious actuators, capable of functioning in both dry and aqueous environments. ${ }^{13}$ Unlike photothermal agents, photomechanical dyes can generate actuation in LC networks with discontinuous as well as continuous alignments, including planar. By generating a gradient in the network pull effect though the polymer's thickness, an effective strain gradient is created which in turn causes actuation, see Fig. 3B(ii). Additionally, actuation of LCPs containing photomechanical azobenzene dyes may produce intermediate deformation states which are temporarily stable, even upon removal of illumination, as the photomechanical dyes remain in light-induced cis state, until the dyes are returned to its initial thermodynamically stable trans state. Summarizing, light responsivity in LCPs can be achieved by the inclusion of either photothermal or photomechanical-based photoswitches, with the nature of the dye determining the actuation characteristics of the resulting material. For azobenzenes chromophores, both the mechanistic pathway for actuation as well as characteristics including 
isomer lifetimes and absorption spectra can be tailored by modifying the chemistry of the photoswitch. ${ }^{18}$

\section{Light-driven soft robots based on LCPs}

Inspired by the locomotive ability of animals, initial developments in LCP light-driven soft robots has concentrated in the realization of devices capable of walking. Single component LC-soft robots with locomotive ability in dry environment include works in which the motion emulates animals such as caterpillars, ${ }^{19-21}$ snails, ${ }^{22}$ and inchworms ${ }^{23}$ Fig. 4. Most of these actuators use photothermal dyes, which upon light absorption heat the polymer network to above the nematic to isotropic transition temperature, triggering fast and local contraction. The anisotropic contraction can then be utilized to power locomotion, Fig. 4A, B, D and E. Other examples utilize local alignment patterns to induce area selective motion without requiring localized illumination, Fig. 4F. Soft robots containing photomechanical chromophores require illumination with two different wavelengths to activate unbending and bending action in an inchworm design for example, Fig. 4C. However, the locomotion freedom of such devices remains limited, being either only in the forwards direction, or forwards and backwards. An example of single component LC device displays the ability to turn, yet even this approach remains a slow process requiring precise illumination to trigger the lateral area of the inchworm design, Fig. 4B. Further locomotion examples demonstrate other modes, including rolling ${ }^{24,25}$ or wave-like motion. ${ }^{18}$ Further research has focused on mimicking the object handing ability of natural organisms by fabricating soft grippers, ${ }^{26}$ making it possible to combine wirelessly controlled gripping ability with untethered locomotion.
Light-driven soft robots are not restricted to dry environments, although those operating underwater are rarely reported, Fig. 5. Initial studies demonstrate LCE microrobots able to swim, powered by a scanning light source. ${ }^{29}$ Actuators operating at the water-air interface include single LCE films that swim into the dark ${ }^{30}$ and a polyimide/LCN bilayer that moves with motion that mimics a dolphin, ${ }^{31}$ Fig. $5 \mathrm{~B}$ and C, respectively. Near infrared (NIR)-driven swimming near the water-air interface driven by reversible bending and unbending motion is also shown with a polydopamine-coated LCE. ${ }^{32}$ Recent developments report on a LC gel containing photothermal chromophores which operates solely underwater and demonstrates light-driven locomotion and swimming upon irradiation with high intensity lasers, Fig. 5A. ${ }^{14}$ The first demonstration of an amphibian-like light-driven LCP actuator has an inchworm inspired design ${ }^{23}$ having one pointed and one flat edge, and can be made to slowly walk on a dry paper surface as well as when the environment is flooded with water, Fig. 5D. ${ }^{13}$ By containing photomechanical azobenzene photoswitches, UV light illumination causes the film to unbend and blue light triggers the reverse actuation. Alternating illumination, directed from the top fuels forward locomotion.

\section{Advanced light-responsive LCP actuators}

Progress in LC-based soft robots is dependent on the development of photoresponsive LC actuators with advanced functionality, such as broadened actuation modes, as well as versatility and accuracy in the control of the deformations. This section presents advancements in light-responsive LC-based polymers

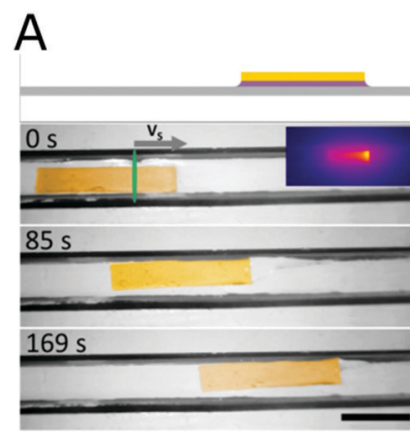

B
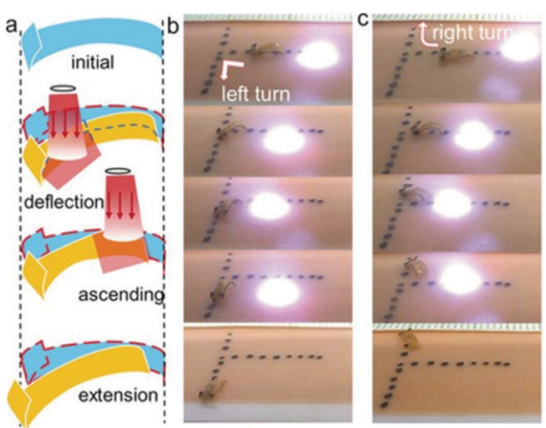

$\mathrm{D}$
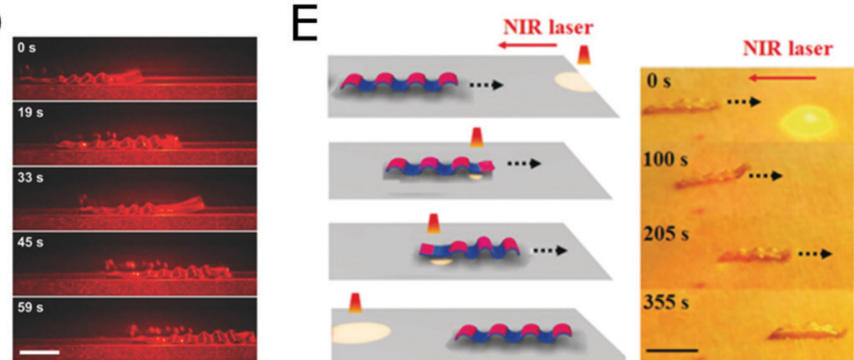

$\mathrm{F}$

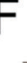

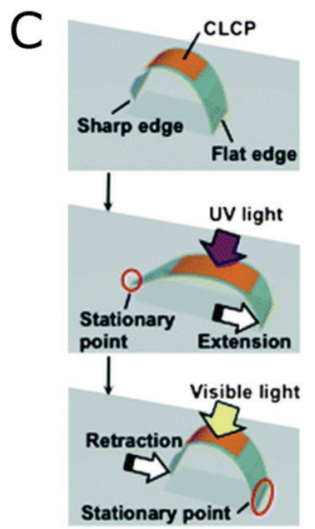

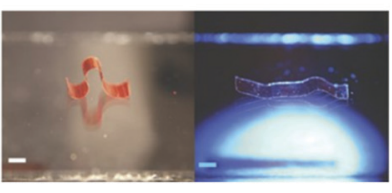

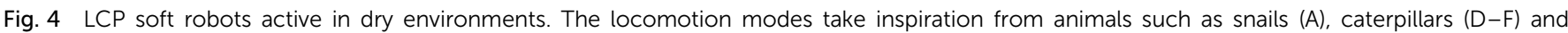

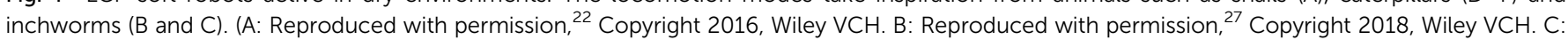

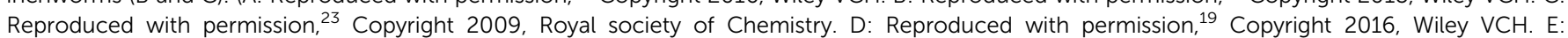
Reproduced with permission, ${ }^{28}$ Copyright 2019, Wiley VCH. F. Reproduced with permission, ${ }^{20}$ Copyright 2017 , Wiley VCH.) 


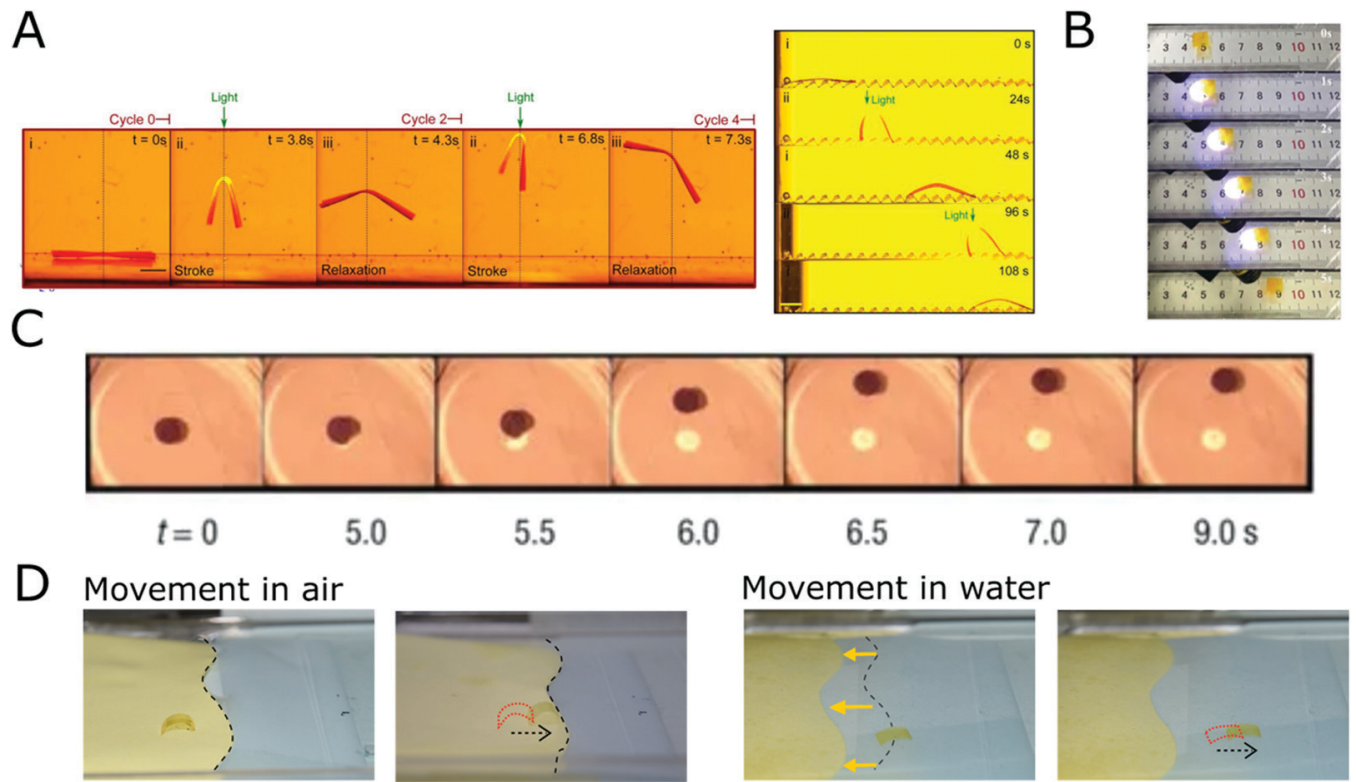

Fig. 5 Examples of light-responsive LC-based soft robots active in aqueous environments ( $\mathrm{A}$ and $\mathrm{D}$ ) or at the air-liquid interface (B and C), mimicking a variety of locomotion modes observed in nature. (A) A liquid crystal gel operating in aqueous environment. Upon irradiation with a laser the film shows locomotion on a ratchet surface or jellyfish-like swimming. Reproduced with permission, ${ }^{14}$ Copyright 2020, National Academy of Sciences. (B) An LCN film is made to move on the water surface upon irradiation, the motion mimics a dolphin. Reproduced with permission, ${ }^{31} \mathrm{Copyright} 2019$, Wiley VCH. (C) An LCE film that moves into the dark on the air/liquid interface. Reproduced with permission, ${ }^{30}$ Copyright 2004, Nature Publishing Group. (D) An amphibian-like inchworm LCN film, showing light-driven locomotion in a dry environment and underwater. Reproduced with permission, ${ }^{13}$ Copyright 2019, Royal society of Chemistry.

concerning continuous motion upon using a constant stimulus and programmed actuator geometries and actuation modes.

\subsection{Shape programmability in light-responsive LCPs}

Unlike natural systems which have complex geometrical shapes and multiple actuation modes, synthetic light-driven soft actuators are often limited in their starting geometry, due to the common fabrication method using flat glass cells. The resulting LCP films usually start flat with a fixed molecular alignment, and are restricted to just one mode of actuation, such as simple bending, Fig. 6. Furthermore, synthetic actuators generally are restricted to flat starting shapes. Developments exploring shape and motion programmability would greatly benefit the freedom in design of LC-based soft robots. A challenge in shape programmability of LC-based actuators is the covalent crosslinking nature of the networks which prevent recyclability of the polymer into different starting geometries. The ability to shape fix the starting geometry of the LCP into any arbitrary state and subsequently trigger reversible actuation through exposure to light remains largely unexplored. Aside from the approach of using dynamic covalent bonds within LCNs ${ }^{33}$ which have poor long-term functionality, a few recent developments strive to achieve shape and motion programmability, Fig. 6. One approach utilizes light of specific wavelengths to encode the type of deformation in LCNs by combining photothermal and photomechanical dyes in the polymer film, achieving photo-rewritable programming of light-driven actuation, Fig. $6 \mathrm{~A} .{ }^{34}$ Another work utilizes light to configure the stimuli-triggered deformation of an anthracene-dimer crosslinked LCN, Fig. 6B. ${ }^{28}$ By photodecrosslinking (possible through photocleavage of the anthracene dimers), the actuator can be selectively patterned with crosslinked (active) and uncrosslinked (inert) regions. Upon heating the polymer or illumination with NIR which is absorbed by the photothermal dyes in the network, the material undergoes reversible actuation dictated by the photopatterned crosslink density regions. Additionally, LCNs containing azomerocyanine photo-switches have been presented as rewritable, dual light-responsive actuators. ${ }^{35}$ By locally changing the absorbance of the chromophore through acid treatments, the films can be designed to have area-specific 'hinges', with each area responding to specific wavelengths of light depending to the chromophore species at that location, Fig. 6C. Yet, even such innovative developments do not alleviate the restrictions of the flat starting geometry of the actuators. Manufacturing techniques such as 3D printing may alleviate restrictions in actuator design, allowing for fabrication of LC-based actuators with alternative initial shapes. ${ }^{36}$ Whereas thermally responsive LC-based devices have been fabricated with 3D printing, no 3D printed light-responsive LC actuators have yet been documented.

Ikeda and co-workers demonstrated an LC-based photoactuator using dynamic covalent bonds whose initial geometry could be designed and subsequently actuated by light, Fig. 6D, yet recyclable shape programming was not demonstrated. ${ }^{37}$ The photoactuator uses a polysiloxane backbone-LCE doped with azobenzene moieties; shape fixing is possible through exchange links between the ester crosslinks and hydroxy groups in the network. More recently, a bilayer composed of a polyethylene terephthalate (PET) spray-coated with an LCN was 


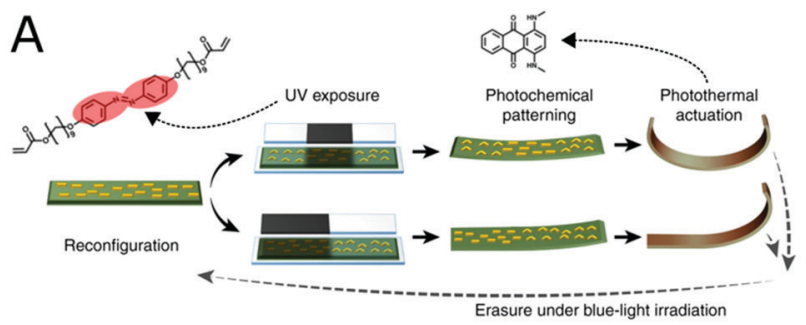

B

C

Erasure under blue-light irradiation
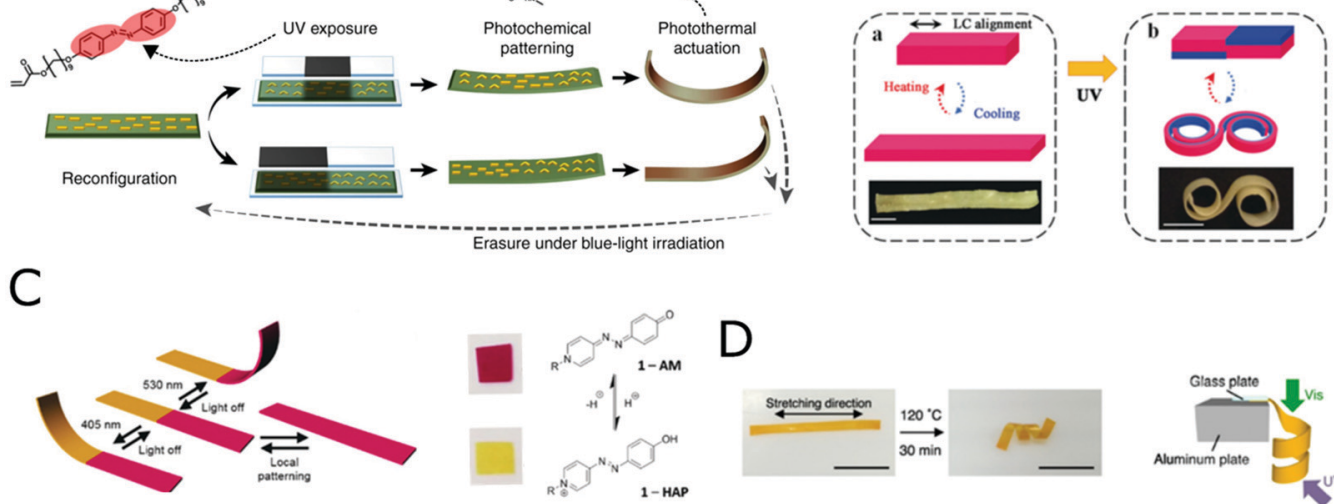

D
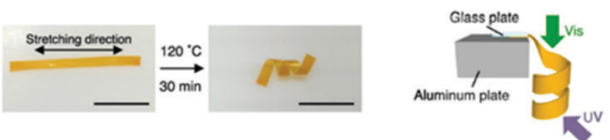

E
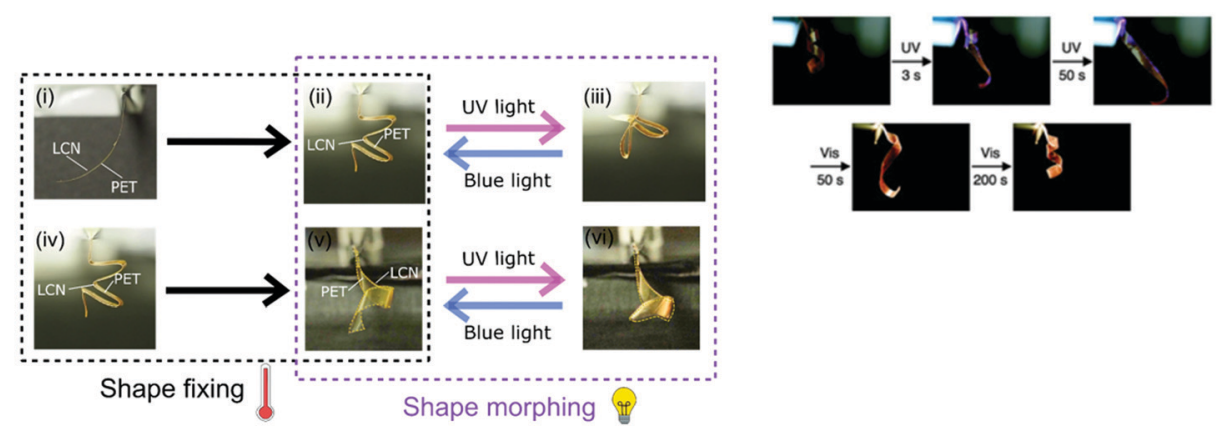

Fig. 6 Examples of shape programming in LCPs. (A) Actuator doped with two types of azobenzene chromophores (one photothermal dye and one photomechanical azobenzene derivative), shows different deformations to the same applied stimulus. The shape deformation is dependent on a programming step which uses light to convert crosslinker-azobenzene dye in the network from the trans to cis states. Reproduced with permission, ${ }^{34}$ Copyright 2018, Nature Publishing Group. (B) A reconfigurable LC actuator. Through selective decrosslinking, the polymer film can be patterned with active and inert regions. Actuation is then possible by heating the material or by exposure to the NIR light, which is absorbed by photothermal dyes in the network. Reproduced with permission, ${ }^{28}$ Copyright 2020, Wiley VCH. (C) A rewritable LCN actuator in which the active areas can be designed with an acid programming step of the photothermal azobenzene, changing its light absorption peak. The independent sections on the actuator respond to different wavelengths of light. Reproduced with permission, ${ }^{35}$ Copyright 2017, Wiley VCH. (D) A light-responsive actuator with programmable shape geometries. The actuator can be programmed into a specific shape through a thermal shaping process and subsequently actuated by light. Reproduced with permission, ${ }^{37}$ Copyright 2016, Wiley VCH. (E) A bilayer actuator demonstrating both shape and motion programmability. The actuation is composed of a PET substrate, spray-coated with a light-responsive LCN. Shape fixing is realized by the thermoplastic PET and light-driven actuation is carried out by the LCN at room temperature. Reproduced with permission, ${ }^{38}$ Copyright 2020 , Wiley VCH.

shown to achieve shape and motion programmability as well as recyclability, Fig. $6 \mathrm{E} .{ }^{38}$ Shaping the actuator above the $T_{\mathrm{g}}$ of the polymers allows for shape fixing ability, due to the thermoplastic nature of the substrate, and subsequent photoactuation, due to the azobenzene chromophores in the LCN, results in a programmed photo deformation.

\subsection{Sustained motion in light-responsive LCPs}

Continual motions, like the heartbeat, are inspirations for the development of soft materials with sustained actuation. Continuous sustained motion in polymers could free devices from depending on on/off switching of external stimuli. Of special interest is the utilization of oscillatory motion to fuel locomotion in future LCP soft robots, increasing locomotion speed and facilitating control. Continual motion in polymer actuators are often a result of feedback loops that couple material properties and external stimuli in synchronization. Light-driven oscillation of liquid crystal cantilevers upon exposure to constant high intensity light has been demonstrated. ${ }^{27,39-44}$ The mechanism for such oscillations commonly relies on the fast photothermal deformation of the LCP to move the material into and out of the light beam, with a feedback loop sustaining the motion. Such work has pioneered the development of devices displaying continual locomotion ${ }^{18,24}$ or rotational, mill-like motion ${ }^{45}$ Fig. 7. In Fig. 7A, a spiral shaped LCN is made to move over a surface by illumination directed from the top. Rolling commences when the spiral sections exposed to light actuate and cause the spiral to roll, this movement exposes a different area of the spiral to the light source which then also actuates, continuing the rolling motion. Feedback loops involving self-shadowing have also been used to trigger movement of an LCN attached to an inert plastic frame, Fig. 7B. Upon exposure to a light, incident at an angle, the LCN initiates a wave-like motion and the contact between the film and the surface below causes the device to move either forwards or backwards, depending on the setup. ${ }^{18}$ The same LCNs have also been employed to realize a milllike motion, Fig. 7C, in which illumination from the side, triggers the actuation of an individual film causing it to tip the mill and place another in the light's focus, setting in motion the feedback loop for the rotating motion. 

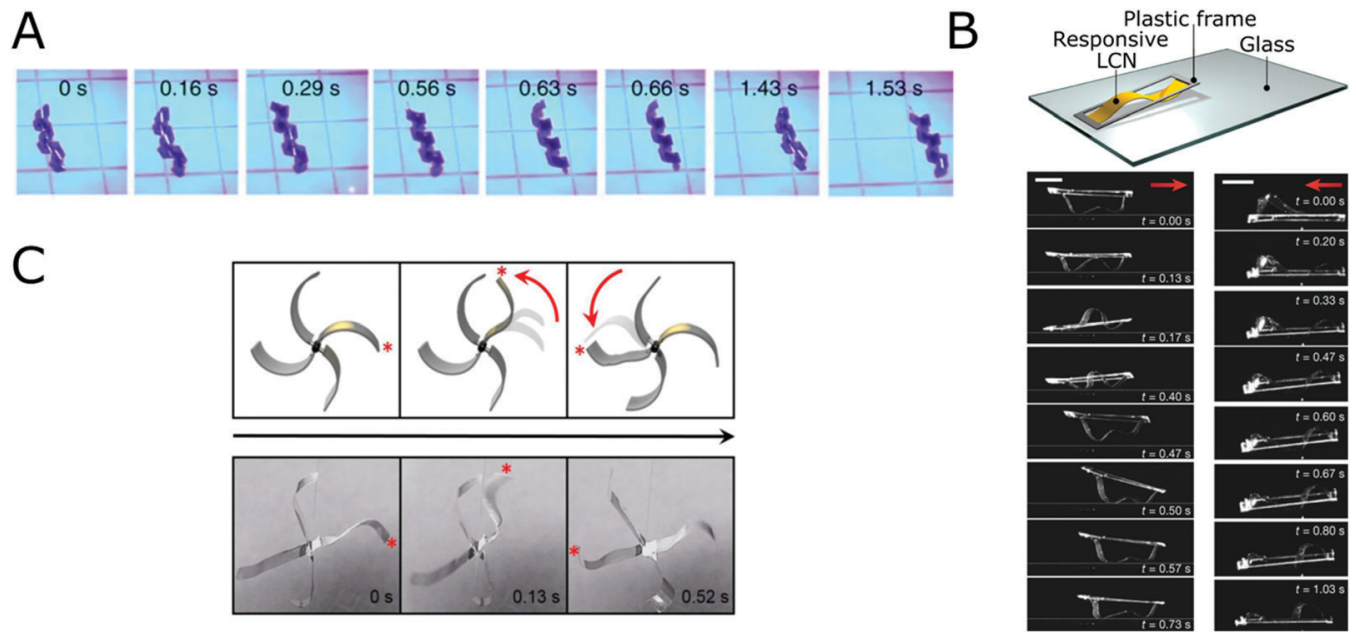

Fig. 7 Sustained motion in LCPs have been utilized in a variety of devices. (A) Photo motility in a spiral LCP. Constant illumination causes the strip to roll, the mechanism of the motion being based on self-shadowing. Reproduced with permission, ${ }^{24}$ Copyright 2016, Nature Publishing Group. (B) An LCN is fixed to a plastic frame. Upon illumination from an angle, the polymer film undergoes a wave-like motion, based on self-shadowing, and causes the whole device to move. Reproduced with permission, ${ }^{18}$ Copyright 2017, Nature Publishing Group. (C) LCN films fixed to a small tube can be made to turn. The motion of each film unbalances the device and causes it to rotate, placing another film in light's focus, initiating a feedback loop that sets the device into a motion resembling a mill. Reproduced with permission, ${ }^{45}$ Copyright 2017, Elsevier.

\section{From single actuators to bioinspired actuator assemblies}

Animals utilize constructive actuation of individual muscles to achieve macroscopic motion and to complete tasks. As previously mentioned, the vast majority of light-driven soft robots have focused on devices based on single actuators: complex multifunctionality or multi-tasking is rarely presented. The realization of orchestrated motion in multi-actuator systems will increase the number of tasks which stimuli-responsive devices are be able to perform. Such assemblies will utilize integrated actuators working in concert to perform tasks such as the integration of locomotion and object handling. Actuator assemblies can be composed of a collection of actuators that respond to the same stimulus, or a variety of soft actuators composed of different materials that respond to different stimuli. Assemblies may also refer to devices taking inspiration from organisms displaying motion at different length scales, including macroscopic motion as well as individual motion of microstructures located on the larger actuator. Even though several works report microscale actuation of LCP materials, ${ }^{46}$ the demonstration of hierarchical materials with both responsive microstructures, such as pillars, on an active substrate, remains unreported for light-driven liquid crystalline systems.

The potential of actuator assemblies has already been demonstrated; see Fig. 8A-D. Actuator assemblies have been utilized to create arm-like motion, with independent actuators functioning as muscle groups in a human arm, Fig. $8 \mathrm{~A}$ and $\mathrm{B} .{ }^{21,47}$ By actuating the different sections in the device, an object can be grasped and lifted (Fig. 8A) or displaced transversely (Fig. 8B). The actuation mechanism follows from the photothermally driven contraction of the LCE, causing the independent sections to actuate individually, such as initial contraction of the arm followed by the gripper section, Fig. 8A. The different segments composing the soft robots in Fig. 8 are commonly assembled with simple methods including glue or adhering tape. Other examples successfully integrate locomotion with object handling ability. A light-powered LCN based soft swimmer inspired by flagella-like motion shows how actuator assemblies can realize underwater devices capable of multitasking, combining a swimming locomotion mode with object handing, Fig. 8C. ${ }^{48}$ This device relies on alternating UV and blue light illumination to activate the photomechanical azobenzene dopant to trigger opening or closing of the gripper or flagella like movement of the tail. Recently, a fully light-driven transporter robot, operating in dry environment, was shown to have both freedom in locomotion as well as ability to pick up, transport and deliver a small cargo, Fig. $8 \mathrm{D} .{ }^{49}$ The device is composed of multiple splay aligned LCP films and utilizes rapid photothermal response to cause unbending of its independent segments. An illumination sequence of four steps is used to complete stride in any direction. By utilizing different chromophores in the device's legs and arms, the soft robot can be wirelessly controlled to walk around corners and selectively deliver the transported cargo. Recent work also presents an artificial coral polyp, composed of two stimuli-responsive materials: a stem made of magnetic response polydimethylsiloxane (PDMS) composite and a light-responsive LCP gripper. ${ }^{50}$ The device demonstrates how different motions of two stimuli-responsive materials present in a single device may be harnessed to perform advanced tasks: by means of a magnetic field, which induces bending of its flexible stem, the device can induce flow in a liquid to attract suspended targets, subsequent capture of the target is realized with its photoresponsive gripper.

\section{Future needs}

Since the first report on azobenzene-doped LCNs showing lightdriven macroscopic contraction of LCEs, by Finkelmann in $2001^{51}$ and bending of LCNs, by Ikeda in $2003,{ }^{52}$ the functionality 

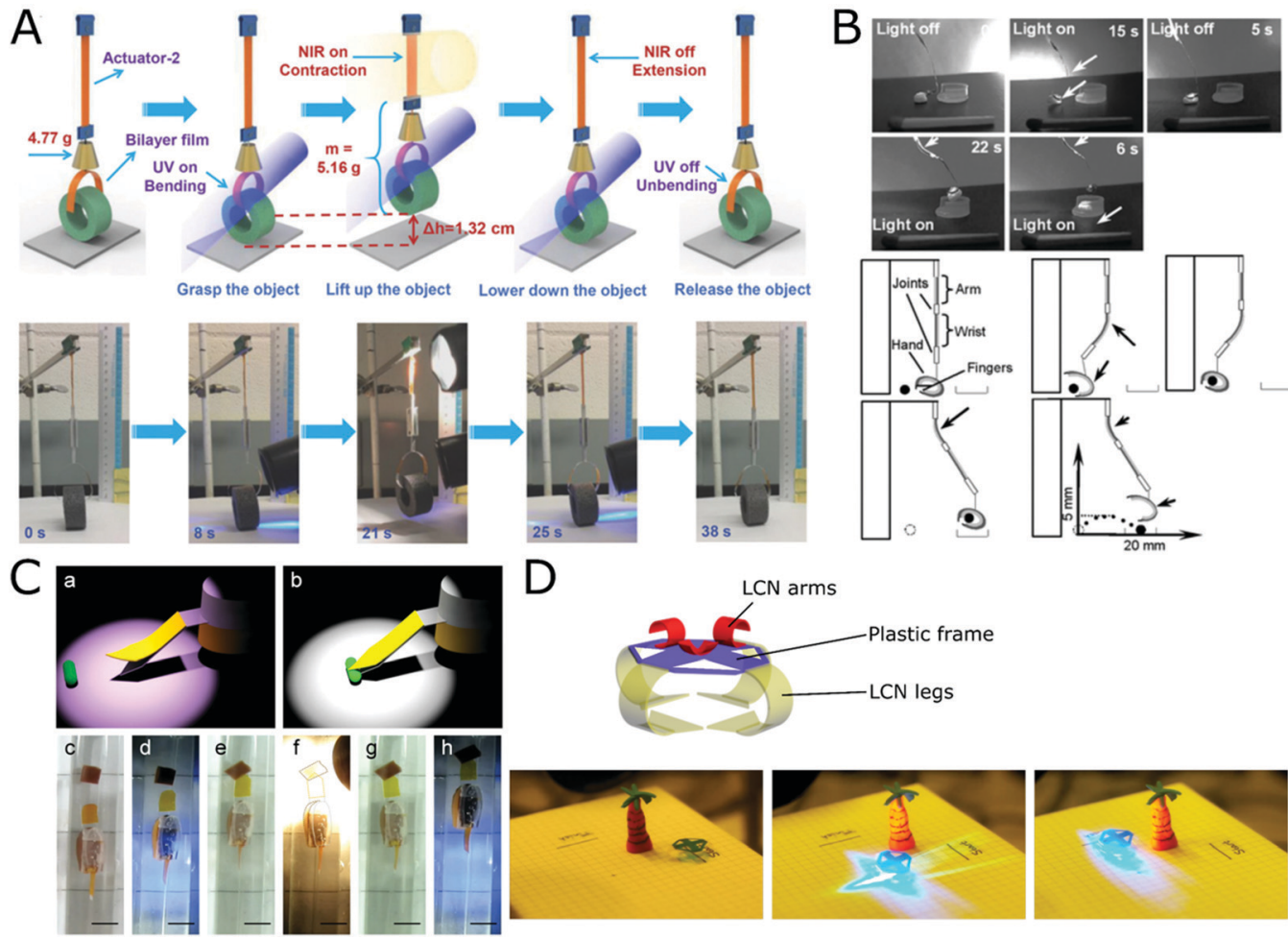

Fig. 8 Actuator assemblies. (A) An actuator assembly mimicking a human arm. The assembly consists of two LCE segments, both actuating via NIR light. Reproduced with permission, ${ }^{21}$ Copyright 2018 , Wiley VCH. (B) A three-component light-driven assembly resembling an arm, demonstrating the ability to perform object pick up and manipulation. Reproduced with permission, ${ }^{47}$ Copyright 2010, Royal society of Chemistry. (C) An LCN-based swimmer. The assembly contains a gripper device at its front and is connected to the device's static central section having a long LCN film acting as a tail which propels the device forward. Both the gripper and the tail are photoactive. Reproduced with permission, ${ }^{48}$ Copyright 2015, Nature Publishing Group. (D) A soft transporter robot capable of moving in multiple directions as well as picking up, transporting and delivering cargo in dry environment. Reproduced with permission, ${ }^{49} \mathrm{Copyright} 2018$, Wiley VCH.

of light-driven LCPs actuator and soft robotics has progressed significantly. However, there still remains several aspects to be addressed before the potential of LCP devices can be fully realized. Among the main challenges seen by the authors are aspects concerning the versatility of the devices, which should strive for amphibian activity, increasing mechanical robustness and development of novel chromophores to overcome the typical dependence on UV light and strive for multi-wavelength responsivity. To increase locomotion versatility in amphibian soft robots, novel designs should consider alternative modes of movement, expanding from walking on land or at the bottom of an aqueous container, towards swimming. Furthermore, the integration of multiple stimuli-responses in a single devices will be advantageous, so that a single segment in the device may be orthogonally triggered by different stimuli to undergo distinct modes of motion, such as bending with one and turning/twisting with the other. ${ }^{53}$ Alternatively, variations of a single stimulus, light, to activate different sections of the actuators can be explored: by using different wavelengths of light bending at different 'hinges' in a polymer device, as has been demonstrated by single actuators. ${ }^{35,54}$

The expansion towards autonomous soft robots calls for actuator assemblies which can communicate and sustain feedback loops for continuous motion. Multi-tasking soft robots will also require actuators capable of performing large amount of work, calling for further development in novel photoswitches and polymer matrices. An additional function which will increase autonomy, is the capability of devices to recognize other objects, as exemplified by an LC-based artificial flytrap which only grasps objects of a certain color..$^{55}$ Furthermore, future developments will expand from current cell-based LCP fabrication methods to upcoming technologies such as 3D printing, which allow for greater freedom in actuator dimensions and provide an easier method to combine actuator assemblies. However, additive manufacturing also raises challenges including the poor mechanical robustness of soft LCEs currently printed and attaining sufficient light penetration to realize actuation in larger 3D printed structures. Future research pursuits may include realization of hierarchical actuators in which motion is induced on multiple length scales, such as micropillars on a centimeter size film. The integration of such hierarchical actuators in selected areas of soft robots will allow for increased versatility by controlling the adhesion of the device with a surface, for example. Such developments may realize climbing robots in which microstructures control attachment of the robot's leg to a wall.

\section{Conclusions}

The potential impact of soft robotics has highlighted the promise of liquid crystal-based polymers in the realization of 
fast, untethered control of active segments in soft robots. The use of LC materials grants unprecedented freedom in design, spanning from the molecular level, involving tunability of ordered mesophases, and control of molecular alignments to the variety of attainable macroscopic actuation modes. Advancements in mechanistic understanding of photoswitches has allowed for the evolution of devices active in water and/or air. Also important have been the developments in actuator functionality, with advanced abilities such as mechanical robustness, sustained motion and multi-stimuli response fueling the debut of novel untethered soft robots progressing towards autonomy and versatility. In turn, the bioinspired construction of light-driven soft robots based on actuator assemblies has only began to demonstrate its potential, resulting in devices with locomotion freedom and ability for multi-tasking. Future progress calls for continued expansion in actuator functionality as well more complex assemblies in which individual segments can influence and communicate with each other. It is with the combined expertise from chemists, material scientists and engineers that the potential of light-driven soft robots in fields such as biomedicine and microfluidics will be uncovered.

\section{Conflicts of interest}

There are no conflicts to declare.

\section{Acknowledgements}

The authors would like to acknowledge the many discussions and contributions of our former and current colleagues. The research of Marina Pilz da Cunha has been supported by the Impuls 2 program of the Eindhoven University of Technology.

\section{References}

1 S. Won, S. Kim, J. E. Park, J. Jeon and J. J. Wie, On-demand orbital maneuver of multiple soft robots via hierarchical magnetomotility, Nat. Commun., 2019, 10, 4751.

2 M. Sitti and D. S. Wiersma, Pros and Cons: Magnetic versus Optical Microrobots, Adv. Mater., 2020, 32, 1906766.

3 T. J. W. Joselle, M. McCracken and B. R. Donovan, Materials as Machines, Adv. Mater., 2020, 32, 1906564.

4 X. Pang, J. Lv, C. Zhu, L. Qin and Y. Yu, Photodeformable Azobenzene-Containing Liquid Crystal Polymers and Soft Actuators, Adv. Mater., 2019, 31, 1904224.

5 C. Majidi, Soft-Matter Engineering for Soft Robotics, Adv. Mater. Technol., 2019, 4, 1800477.

6 G. Stoychev, A. Kirillova and L. Ionov, Light-Responsive Shape-Changing Polymers, Adv. Opt. Mater., 2019, 7, 1900067.

7 T. J. White and D. J. Broer, Programmable and adaptive mechanics with liquid crystal polymer networks and elastomers, Nat. Publ. Gr., 2015, 14, 1087-1098.

8 J. Kupfer and H. Finkelmann, Nematic liquid single crystal elastomers, Makromol. Chem., Rapid Commun., 1991, 12, 717-726.
9 D. J. Broer and G. N. Mol, Anisotropic thermal expansion of densely cross-linked oriented polymer networks, Polym. Eng. Sci., 1991, 31, 625-631.

10 C. Ohm, M. Brehmer and R. Zentel, Liquid crystalline elastomers as actuators and sensors, Adv. Mater., 2010, 22, 3366-3387.

11 H. K. Bisoyi and Q. Li, Light-Driven Liquid Crystalline Materials: From Photo-Induced Phase Transitions and Property Modulations to Applications, Chem. Rev., 2016, 116, 15089-15166.

12 L. Dong and Y. Zhao, Photothermally driven liquid crystal polymer actuators, Mater. Chem. Front., 2018, 2, 1932-1943.

13 M. Pilz da Cunha, E. A. J. van Thoor, M. G. Debije, D. J. Broer and A. P. H. J. Schenning, Unravelling the photothermal and photomechanical contributions to actuation of azobenzene-doped liquid crystal polymers in air and water, J. Mater. Chem. C, 2019, 7, 13502-13509.

14 H. Shahsavan, A. Aghakhani, H. Zeng, Y. Guo, Z. S. Davidson, A. Priimagi and M. Sitti, Bioinspired underwater locomotion of light-driven liquid crystal gels, Proc. Natl. Acad. Sci. U. S. A., 2020, 117, 5125-5133.

15 S. X. A. N. P. Rochon, Recent Developments in Aromatic Azo Polymers Research, Chem. Mater., 1993, 5, 403-411.

16 K. M. Lee, N. V. Tabiryan, T. J. Bunning and T. J. White, Photomechanical mechanism and structure-property considerations in the generation of photomechanical work in glassy, azobenzene liquid crystal polymer networks, J. Mater. Chem., 2012, 22, 691-698.

17 J. H. Yun, C. Li, S. Kim and M. Cho, Comparing Photoactuation of an Azobenzene-Doped Nematic Liquid Crystal Polymer through Its Activation Mechanism: Trans-Cis-Trans Reorientation and Photoisomerization, J. Phys. Chem. C, 2018, 122, 6310-6317.

18 A. H. Gelebart, D. J. Mulder, M. Varga, A. Konya, G. Vantomme, E. W. Meijer, R. L. B. Selinger and D. J. Broer, Making waves in a photoactive polymer film, Nature, 2017, 546, 632-636.

19 M. Rogóż, H. Zeng, C. Xuan, D. S. Wiersma and P. Wasylczyk, Light-Driven Soft Robot Mimics Caterpillar Locomotion in Natural Scale, Adv. Opt. Mater., 2016, 4, 1689-1694.

20 H. Zeng, O. M. Wani, P. Wasylczyk and A. Priimagi, LightDriven, Caterpillar-Inspired Miniature Inching Robot, Macromol. Rapid Commun., 2017, 39, 1700224.

21 X. Lu, H. Zhang, G. Fei, B. Yu, X. Tong, H. Xia and Y. Zhao, Liquid-Crystalline Dynamic Networks Doped with Gold Nanorods Showing Enhanced Photocontrol of Actuation, Adv. Mater., 2018, 30, 1706597.

22 M. Rogóż, K. Dradrach, C. Xuan and P. Wasylczyk, A Millimeter-Scale Snail Robot Based on a Light-Powered Liquid Crystal Elastomer Continuous Actuator, Macromol. Rapid Commun., 2019, 40, 1900279.

23 M. Yamada, M. Kondo, R. Miyasato, Y. Naka, J. I. Mamiya, M. Kinoshita, A. Shishido, Y. Yu, C. J. Barrett and T. Ikeda, Photomobile polymer materials - Various three-dimensional movements, J. Mater. Chem., 2009, 19, 60-62.

24 J. J. Wie, M. R. Shankar and T. J. White, Photomotility of polymers, Nat. Commun., 2016, 7, 13260. 
25 Y. C. Cheng, H. C. Lu, X. Lee, H. Zeng and A. Priimagi, Kirigami-Based Light-Induced Shape-Morphing and Locomotion, Adv. Mater., 2019, 32, 1906233.

26 C. K. Yoon, Advances in biomimetic stimuli responsive soft grippers, Nano Convergence, 2019, 6, 20.

27 F. Ge, R. Yang, X. Tong, F. Camerel and Y. Zhao, A Multifunctional Dye-doped Liquid Crystal Polymer Actuator: LightGuided Transportation, Turning in Locomotion, and Autonomous Motion, Angew. Chem., Int. Ed., 2018, 57, 11758-11763.

28 Z. C. Jiang, Y. Y. Xiao, X. Tong and Y. Zhao, Selective Decrosslinking in Liquid Crystal Polymer Actuators for Optical Reconfiguration of Origami and Light-Fueled Locomotion, Angew. Chem., Int. Ed., 2019, 58, 5332-5337.

29 S. Palagi, A. G. Mark, S. Y. Reigh, K. Melde, T. Qiu, H. Zeng, C. Parmeggiani, D. Martella, A. Sanchez-Castillo, N. Kapernaum, F. Giesselmann, D. S. Wiersma, E. Lauga and P. Fischer, Structured light enables biomimetic swimming and versatile locomotion of photoresponsive soft microrobots, Nat. Mater., 2016, 15, 647-653.

30 M. Camacho-Lopez, H. Finkelmann, P. Palffy-Muhoray and M. Shelley, Fast liquid-crystal elastomer swims into the dark, Nat. Mater., 2004, 3, 307-310.

31 S. Ma, X. Li, S. Huang, J. Hu and H. Yu, A Light-Activated Polymer Composite Enables On-Demand Photocontrolled Motion: Transportation at the Liquid/Air Interface, Angew. Chem., Int. Ed., 2019, 58, 2655.

32 H. Tian, Z. Wang, Y. Chen, J. Shao, T. Gao and S. Cai, Polydopamine-Coated Main-Chain Liquid Crystal Elastomer as Optically Driven Artificial Muscle, ACS Appl. Mater. Interfaces, 2018, 10, 8307-8316.

33 Z. C. Jiang, Y. Y. Xiao and Y. Zhao, Shining Light on Liquid Crystal Polymer Networks: Preparing, Reconfiguring, and Driving Soft Actuators, Adv. Opt. Mater., 2019, 7, 1900262.

34 M. Lahikainen, H. Zeng and A. Priimagi, Reconfigurable photoactuator through synergistic use of photochemical and photothermal effects, Nat. Commun., 2018, 9, 4148.

35 A. H. Gelebart, D. J. Mulder, G. Vantomme, A. P. H. J. Schenning and D. J. Broer, A Rewritable, Reprogrammable, Dual LightResponsive Polymer Actuator, Angew. Chem., Int. Ed., 2017, 56, 13436-13439.

36 C. P. Ambulo, J. J. Burroughs, J. M. Boothby, H. Kim, M. R. Shankar and T. H. Ware, Four-dimensional Printing of Liquid Crystal Elastomers, ACS Appl. Mater. Interfaces, 2017, 9, 37332-37339.

37 T. Ube, K. Kawasaki and T. Ikeda, Photomobile LiquidCrystalline Elastomers with Rearrangeable Networks, Adv. Mater., 2016, 28, 8212-8217.

38 R. C. P. Verpaalen, M. Pilz da Cunha, T. A. P. Engels, M. G. Debije and A. P. H. J. Schenning, Liquid Crystal Networks on Thermoplastics: Reprogrammable Photo-Responsive Actuators, Angew. Chem., Int. Ed., 2020, 59, 4532-4536.

39 T. J. White, N. V. Tabiryan, S. V. Serak, U. A. Hrozhyk, V. P. Tondiglia, H. Koerner, R. A. Vaia and T. J. Bunning, A high frequency photodriven polymer oscillator, Soft Matter, 2008, 4, 1796.
40 S. Serak, N. Tabiryan, R. Vergara, T. J. White, R. A. Vaia and T. J. Bunning, Liquid crystalline polymer cantilever oscillators fueled by light, Soft Matter, 2010, 6, 779-783.

41 N. Tabiryan, H. Koerner, K. M. Lee, T. J. White, T. J. Bunning, R. A. Vaia and M. L. Smith, Photodriven, Flexural-Torsional Oscillation of Glassy Azobenzene Liquid Crystal Polymer Networks, Adv. Funct. Mater., 2011, 21, 2913-2918.

42 A. H. Gelebart, G. Vantomme, E. W. Meijer and D. J. Broer, Mastering the Photothermal Effect in Liquid Crystal Networks: A General Approach for Self-Sustained Mechanical Oscillators, Adv. Mater., 2017, 29, 1606712.

43 H. Zeng, M. Lahikainen, L. Liu, Z. Ahmed, O. M. Wani, M. Wang, H. Yang and A. Priimagi, Light-fuelled freestyle self-oscillators, Nat. Commun., 2019, 10, 5057.

44 W. Wei, Z. Zhang, J. Wei, X. Li and J. Guo, Phototriggered Selective Actuation and Self-Oscillating in Dual-Phase Liquid Crystal Photonic Actuators, Adv. Opt. Mater., 2018, 6, 1800131.

45 G. Vantomme, A. H. Gelebart, D. J. Broer and E. W. Meijer, A four-blade light-driven plastic mill based on hydrazone liquid-crystal networks, Tetrahedron, 2017, 73, 4963-4967.

46 F. Ge and Y. Zhao, Microstructured Actuation of Liquid Crystal Polymer Networks, Adv. Funct. Mater., 2019, 30, 1901890.

47 F. Cheng, R. Yin, Y. Zhang, C.-C. Yen and Y. Yu, Fully plastic microrobots which manipulate objects using only visible light, Soft Matter, 2010, 6, 3447.

48 C. Huang, J. A. Lv, X. Tian, Y. Wang, Y. Yu and J. Liu, Miniaturized swimming soft robot with complex movement actuated and controlled by remote light signals, Sci. Rep., 2015, 5, 17414.

49 M. Pilz da Cunha, S. Ambergen, M. G. Debije, E. F. G. A. Homburg, J. M. J. Den Toonder and A. P. H. J. Schenning, A Soft Transporter Robot Fueled by Light, Adv. Sci., 2020, 7, 1902842.

50 M. Pilz, H. S. Kandail, J. M. J. Den Toonder and A. P. H. J. Schenning, An artificial aquatic polyp that wirelessly attracts, grasps, and releases objects, Proc. Natl. Acad. Sci. U. S. A., 2020, 117, 17571-17577.

51 H. Finkelmann, E. Nishikawa, G. G. Pereira and M. Warner, A new opto-mechanical effect in solids, Phys. Rev. Lett., 2001, 87, 015501.

52 B. T. Ikeda, M. Nakano, Y. Yu, O. Tsutsumi and A. Kanazawa, Anisotropic Bending and Unbending Behavior of Azobenzene Liquid-Crystalline Gels by Light Exposure, Adv. Mater., 2003, 15, 201-205.

53 M. Pilz, Y. Foelen, T. A. P. Engels, K. Papamichou, M. Hagenbeek, M. G. Debije and A. P. H. J. Schenning, On Untethered, Dual Magneto- and Photoresponsive Liquid Crystal Bilayer Actuators Showing Bending and Rotating Motion, Adv. Opt. Mater., 2019, 7, 1801604.

54 C. L. Van Oosten, C. W. M. Bastiaansen and D. J. Broer, Printed artificial cilia from liquid-crystal network actuators modularly driven by light, Nat. Mater., 2009, 8, 677-682.

55 O. M. Wani, H. Zeng and A. Priimagi, A light-driven artificial flytrap, Nat. Commun., 2017, 8, 15546. 